\title{
MONTE CARLO SIMULATION OF HALL EFFECT IN n-TYPE GaN
}

\author{
J.D. Albrecht ${ }^{\dagger}$, P.P. Ruden ${ }^{\dagger}$, E. Bellotti ${ }^{\ddagger}$, and K.F. Brennan ${ }^{\ddagger}$ \\ ${ }^{\dagger}$ ECE Department, University of Minnesota, Minneapolis, MN 55455 \\ ${ }^{\ddagger}$ School of ECE, Georgia Institute of Technology, Atlanta, GA 30332
}

\section{Cite this article as: MRS Internet J. Nitride Semicond. Res. 4S1, G6.6 (1999)}

\begin{abstract}
Results of Monte Carlo simulations of electron transport for wurtzite phase GaN in crossed, weak electric and magnetic fields are presented. It is found that the Hall factor, $\mathrm{r}_{H}=$ $\mu_{\text {Hall }} / \mu_{\text {drift }}$, decreases monotonically as the temperature increases from $77 \mathrm{~K}$ to $400 \mathrm{~K}$. The low temperature value of the Hall factor increases significantly with increasing doping concentration. The Monte Carlo simulations take into account the electron-lattice interaction through polar optical phonon scattering, deformation potential acoustic phonon scattering (treated as an inelastic process), and piezoelectric acoustic phonon scattering. Impurity scattering due to ionized and neutral donors is also included, with the latter found to be important at low temperature due to the relatively large donor binding energy which implies considerable carrier freeze-out already at liquid nitrogen temperature. The temperature dependences of the electron concentration, drift mobility, and Hall factor are calculated for donor concentrations equal to $5 \mathrm{x} 10^{16} \mathrm{~cm}^{-3}, \quad 10^{17}$ $\mathrm{cm}^{-3}$, and $5 \times 10^{17} \mathrm{~cm}^{-3}$. The Monte Carlo simulations are compared to classical analytical results obtained using the relaxation-time approximation, which is found to be adequate at low temperatures and sufficiently low carrier concentrations so that inelastic scattering effects due to optical phonons and degeneracy effects are negligible. The influence of dislocations on the Hall factor is discussed briefly.
\end{abstract}

\section{INTRODUCTION}

Electronic characterization of epitaxially grown $\mathrm{GaN}$ relies on temperature dependent Hall measurements to accurately determine carrier concentration and mobility. Analysis of measured Hall data has already led to the examination of several effects unique to GaN Hall measurements. Specifically, the variation of the electron Hall mobility in the presence of a highly conducting layer at the interface between the substrate and the epitaxial GaN crystal has been resolved ${ }^{1}$, and the dependence of the Hall measurement on the orientation of the current with respect to threading dislocations has recently been explained ${ }^{2}$. Also, the low temperature behavior of measured electron mobilities in heavily doped GaN has been found to be consistent with transport associated with band-tailing and impurity-band states ${ }^{3}$. Exceptions aside, interpretations of GaN Hall data often make the assumption that $\mu_{\text {Hall }}=\mu_{\text {drift }}$, independent of temperature ${ }^{4,5}$. One consequence of assuming a unity Hall factor is that total donor concentrations and donor activation energies extracted from electrical measurements are difficult to analyze with confidence. 
We present new calculations of the Hall factor, $r_{H}$, for $n$-type wurtzite $\mathrm{GaN}$ that predict a monotonic decrease with increasing temperature for fixed donor concentrations. The presentation of calculated electron concentration, Hall factor, and drift mobility is followed by a comparison to relaxation-time approximation results and by a discussion of the Hall factor for dislocation dominated scattering.

\section{HALL SIMULATION}

Due to thermal fluctuations in the calculated drift velocity components, calculations of electron drift velocity in crossed electric and magnetic fields using the Monte Carlo method were, until recently, limited to high electric and magnetic field conditions ${ }^{6}$. Advances in computation speed and in the transport simulation algorithm have enabled the application of the Monte Carlo method to weak field conditions. Hall simulations have been reported for a variety of semiconductor materials: $\mathrm{Si}$ and $\mathrm{Ge}^{7}$, InSb and other highly polar semiconductors ${ }^{8}$, and $\mathrm{GaAs}^{9}$. For GaN, electron drift velocities for both large and small static electric fields have been calculated in this manner and low field drift mobility expressions suitable for device modeling have been developed for temperatures above $300 \mathrm{~K}^{10}$.

The present Hall factor calculations use the same approximate GaN analytic band structure which has been published previously ${ }^{10}$, although for the present study of low field transport only the parabolic part of the central conduction band valley is relevant. The electric field strengths are chosen small enough to ensure that ohmic conduction is modeled, and the magnetic field is held in the low field regime such that $\mu_{\text {drift }}|\mathrm{B}|=1.5 \times 10^{-2}$. For instance, at liquid nitrogen temperature and $\mathrm{N}_{\mathrm{D}}=10^{17} \mathrm{~cm}^{-3}$, the electric field in the linear regime is taken to be less than 0.5 $\mathrm{kV} / \mathrm{cm}$ which results in $\mu=2490 \mathrm{~cm}^{2} \mathrm{~V}^{-1} \mathrm{sec}^{-1}$ and, consequently, $|\mathrm{B}|=0.06$ Tesla. The free flight motion of the electrons in crossed electric and magnetic fields is carried out in the usual way for Monte Carlo simulations ${ }^{6}$. The scattering parameters are the same as those in reference 10 and references therein. We favor acoustic deformation potential scattering with energy dissipation over the elastic approximation. This treatment of the acoustic phonon scattering prevents the simulated electron distribution from artificially skewing towards higher energies as would otherwise occur when polar optical phonon scattering is negligible. In addition, neutral impurity scattering is included in our calculations. The effects of neutral impurities become important at low temperatures in material that is not dominated by dislocation scattering.

In Figure 1, the electron concentration is plotted as a function of temperature using

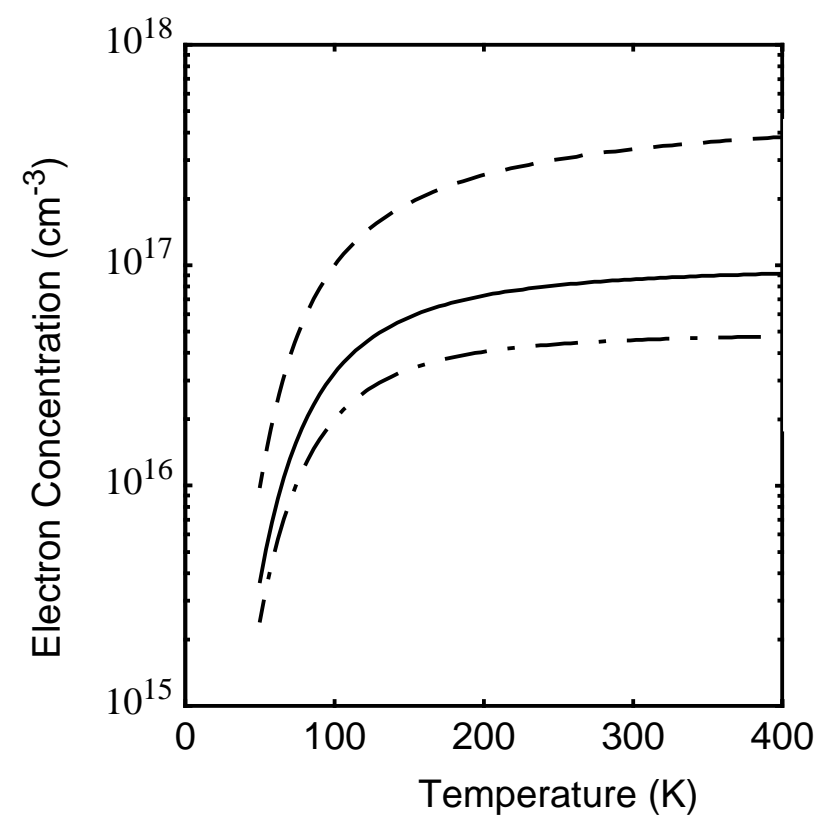

Figure 1: Electron concentration vs. temperature for GaN with donor doping densities of $5 \times 10^{16}$ $\mathrm{cm}^{-3}$ (dash-dotted), $10^{17} \mathrm{~cm}^{-3}$ (solid), and $5 \times 10^{17}$ $\mathrm{cm}^{-3}$ (dashed). 
the screened shallow donor binding energy results of Wang, et al for different background electron concentrations ${ }^{11}$. The temperature effects on the carrier screening are taken into account for this calculation. The figure clearly shows that for uncompensated $\mathrm{GaN}$ at liquid nitrogen temperature there is a large fraction of neutral donor impurities present.

Scattering due to neutral donors is adapted for the Monte Carlo method from the relaxation-time result of Meyer and Bartoli ${ }^{12}$. An analytic expression for the total scattering rate which is valid over the entire energy range of electron energies can be derived from ref. 12 . The neutral impurity scattering rate in terms of the total collision cross section, $\sigma_{c}$, is written as:

$\left.\frac{1}{\tau_{c}}\right|_{\text {neut. imp. }}=N^{x} v \sigma_{c}$

where

$\sigma_{c}=\pi a_{o}^{2}\left(\frac{11.2}{w}\right) \frac{\left(1+e^{-50 w}\right)\left(1+80.6 w+23.7 w^{2}\right)}{\left(1+41.3 w+133 w^{2}\right)}\left\{\left(\frac{w}{6}\right) \frac{\left(12+18 w+7 w^{2}\right)}{(1+w)^{3}}\right\}$,

$N^{x}$ is the concentration of neutral donor scattering centers, $v$ is the electron velocity, $\mathrm{E}_{\mathrm{D}}$ is the donor binding energy, $w=\left(a_{o} k\right)^{2}=\mathrm{E} / \mathrm{E}_{\mathrm{D}}$, and $a_{o}=\mathrm{e}^{2} / 2 \kappa_{\mathrm{o}} \mathrm{E}_{\mathrm{D}}$ which defines an effective Bohr radius. Since scattering of low energy electrons by neutral impurities is velocity randomizing, the collision cross section differs from the momentum-transfer cross section of Meyer and Bartoli ${ }^{12}$ only for incident particles with energies much greater than the donor binding energy. The Born approximation is valid for fast electrons where the term in \{\} of equation (2) is dominant. The collisions are treated as elastic and exchange effects are neglected.

In the Monte Carlo simulation, the final scattering states are determined using the angular distribution derived from the Born scattering amplitude given by ${ }^{13}$

$f_{\text {Born }}(\theta)=-\frac{2}{a_{o} q^{2}}\left[\left(1+\frac{a_{o}^{2} q^{2}}{4}\right)^{-2}-1\right] \quad q=2 k \sin \frac{\theta}{2}$

where $\theta$ is the angle between initial and final k-vectors. It is easily shown that the normalized probability distribution of scattering angles is

$P(\cos \theta)=\frac{\int_{-1}^{\cos \theta}\left|f_{B o r n}(\theta)\right|^{2} d \cos \theta}{\int_{-1}^{+1}\left|f_{B o r n}(\theta)\right|^{2} d \cos \theta}=\frac{[4+w(1-\cos \theta)]^{2}}{[2+w(1-\cos \theta)]^{4}} \frac{6[1+w]^{3}}{\left[12+18 w+7 w^{2}\right]}$

A flat angular distribution results in the low energy $(w \rightarrow 0)$ limit. This is consistent with the velocity randomizing behavior for low electron energy implicit in the phenomenological collision cross section of equation (2). 
Figures 1 and 2 display the calculated free carrier concentrations and electron drift mobilities for fixed doping concentrations. For uncompensated GaN, Figure 1 indicates that the ionized impurity concentration at $77 \mathrm{~K}$ is a small fraction of that at $300 \mathrm{~K}$. In Figure 2 , the temperature dependent drift mobility is plotted for ionized impurity concentrations corresponding to Figure 1. The dashed curves at low temperature indicate the analytical relaxation-time approximation ${ }^{14}$ to the drift mobility including all of the scattering mechanisms except polar optical phonon scattering. The decrease in mobility at low temperature is caused in part by neutral impurity scattering. For the lowest doping concentration considered in this calculation, $\mathrm{N}_{\mathrm{D}}=5 \times 10^{16} \mathrm{~cm}^{-3}$, we find that the neutral impurity scattering plays a large role at low temperature because

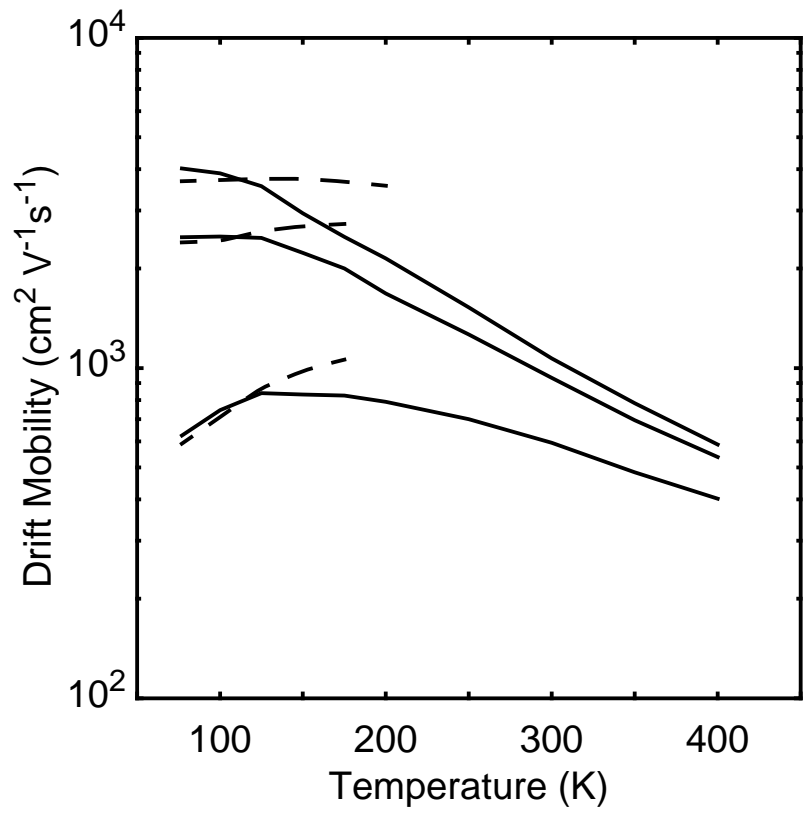

Figure 2: Electron drift mobility vs. temperature for GaN with donor doping densities of $5 \times 10^{16}$ $\mathrm{cm}^{-3}$ (top line), $10^{17} \mathrm{~cm}^{-3}$ (middle), and $5 \times 10^{17}$ $\mathrm{cm}^{-3}$ (lowest). The dashed lines indicate the relaxation-time approximation at low temperature. of the significant carrier freeze-out evident from Figure 1. At 77K, the relaxation-time approximation result for the mobility is $16 \%$ higher than that shown in Figure 2 without the inclusion of neutral impurity scattering effects. The low temperature mobility obtained by Monte Carlo simulation which includes energy dissipation in the acoustic deformation potential scattering is slightly larger than that obtain from the analytical relaxation-time model which uses an elastic approximation.

If the mobility at $77 \mathrm{~K}$ was dominated solely by ionized impurity scattering such that $\mu \sim T^{3 / 2} / n_{\mathrm{I}}$, the decrease in mobility at low temperatures would not be as great or would completely vanish for uncompensated GaN. Conversely, a greater decrease in drift mobility is evident for heavily compensated GaN since the ionized impurity concentration remains substantial at low temperatures ${ }^{15,16}$. Our calculations indicate that having an acceptor concentration of $5 \mathrm{x}$ $10^{16} \mathrm{~cm}^{-3}$ reduces the $77 \mathrm{~K}$ mobility for GaN with $10^{17} \mathrm{~cm}^{-3}$ donors to approximately $800 \mathrm{~cm}^{2}$ $\mathrm{V}^{-1} \mathrm{~s}^{-1}$, or by $30 \%$. Further reductions in mobility from dislocation scattering have been predicted and recently measured by $\mathrm{Ng}$, et $a l^{17}$ for lateral drift normal to charged dislocations in $n$-type GaN films.

The Hall factor determined from our Monte Carlo simulation is plotted in Figure 3 with the error bars indicating the maximum deviation from the average steady-state value. In this context, steady-state was established by simulating $10^{7}$ collisions. The number of collisions was chosen sufficiently large such that the estimated electron drift velocity perpendicular to the applied electric field can be resolved above the thermal fluctuations. Fluctuations in the Monte Carlo drift velocity are inversely proportional to the square-root of the number of collisions simulated. In all three plots of Figure $3, \mathrm{r}_{\mathrm{H}}$ decreases with increasing temperature to a value approaching 1.2. A similar downward trend from 1.5 toward 1.2 at room temperature has been calculated using a variational technique ${ }^{15}$. The increase in $r_{H}$ at low temperatures is due to dom- 
inant impurity scattering. We also expect that $\mathrm{r}_{\mathrm{H}}$ should exhibit a similar trend at low temperature for Hall measurements of mobility perpendicular to the growth direction in samples where scattering from threading dislocations is dominant.

When the mobility is limited solely by dislocation scattering and this process can be modeled by scattering from a line charge, $\mathrm{r}_{\mathrm{H}}$ can be estimated by computing the average momentum relaxation time. It follows from the analytic expression given in reference 2 that,

$$
\mathrm{r}_{\mathrm{H}}=\frac{\left\langle\tau_{\mathrm{m}}^{2}\right\rangle}{\left\langle\tau_{\mathrm{m}}\right\rangle^{2}}=\frac{\pi}{8} \gamma \exp (-1 / \gamma)\left[K_{2}(1 /(2 \gamma))\right]^{-2}\left[8+60 \gamma+210 \gamma^{2}+315 \gamma^{3}\right] ; \gamma=\frac{8 m^{*} \lambda^{2} \mathrm{k}_{\mathrm{B}} \mathrm{T}}{\overline{\mathrm{h}}^{2}}
$$

Here, $K_{2}$ is the second order modified Bessel function and $\lambda$ is the Debye screening length. In Figure $4, \mathrm{r}_{\mathrm{H}}$ as obtained from equation (5) is plotted for drift perpendicular to threading dislocations as a function of temperature. The free carrier density used to determine the screening length is that shown in Figure 1 for uncompensated GaN. The region of interest in Figure 4 is at low temperatures where dislocation scattering may be dominant ${ }^{17}$. Specifically, $\mathrm{r}_{\mathrm{H}}$ is smaller than but close to 1.93 (the $\lambda \rightarrow \infty$ limit). From this estimate, it is reasonable to expect that when electron mobility is dominated by dislocation scattering, the Hall factor will tend to values larger than the room temperature value - perhaps to an extent greater than the Monte Carlo simulations for material without dislocations predict in Figure 3.

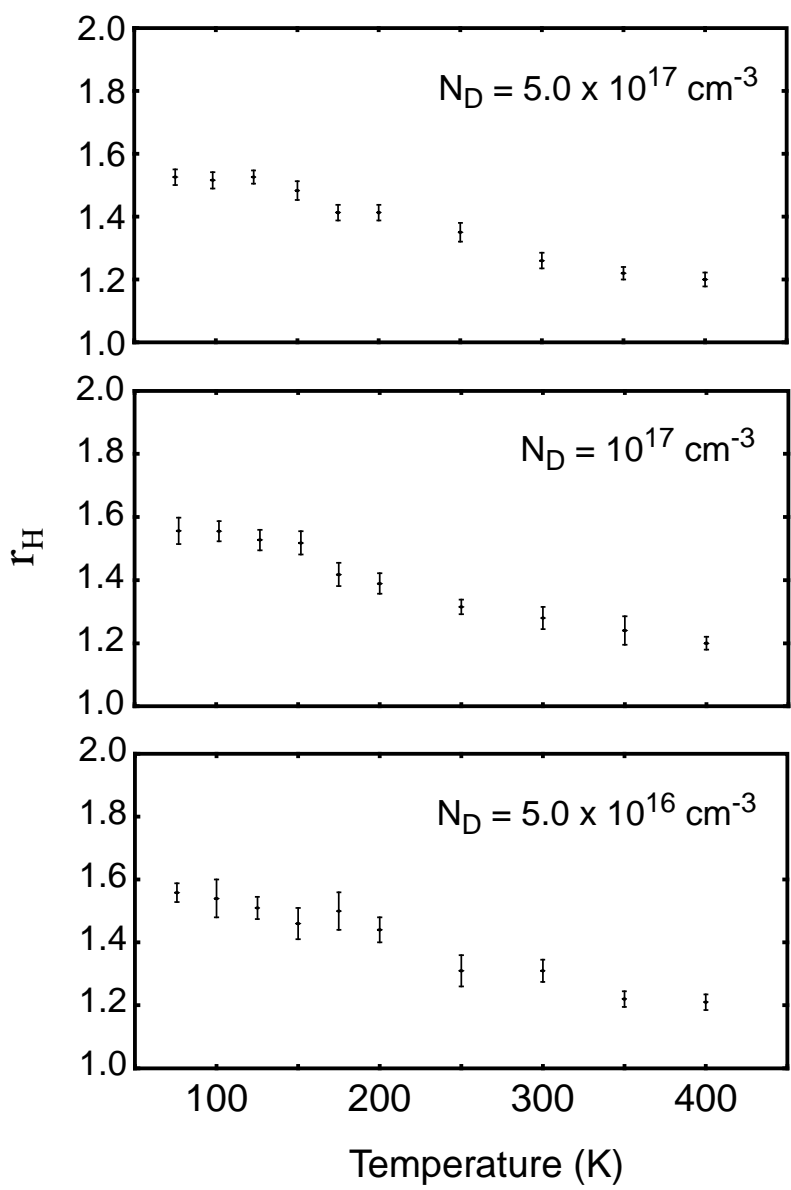

Figure 3: Hall factor vs. temperature for fixed doping concentrations from Monte Carlo simulations.
In summary, Monte Carlo simulations of electron transport in crossed electric and magnetic fields are used to compute mobility and Hall factor values at various temperatures and doping concentrations. This technique is particularly desirable for simulating Hall transport governed by polar optical phonon scattering and we show that $r_{H}$ should be approximately 1.2 for temperatures above $300 \mathrm{~K}$. At lower temperatures $r_{H}$ deviates farther from unity, and the values obtained by simulation are consistent with those obtained from classical analytical approximations. We have also addressed Hall measurements of lateral mobility, which can be dominated by scattering from threading dislocations when dislocation densities are high. In those cases, we predict that $r_{H}$ will remain significantly larger than unity at low temperature, especially for low carrier concentrations where screening is weak. 


\section{ACKNOWLEDGEMENTS}

This work benefited from useful discussions with J.R. Meyer. A grant from the Minnesota Supercomputer Institute is gratefully acknowledged. This work was supported in part by Hughes Research Laboratories and by NSF under grant number ECS-9811366. The work at Georgia Tech was sponsored in part by NORTEL through contract E21-5D7 and by ONR through contract E21-K19.

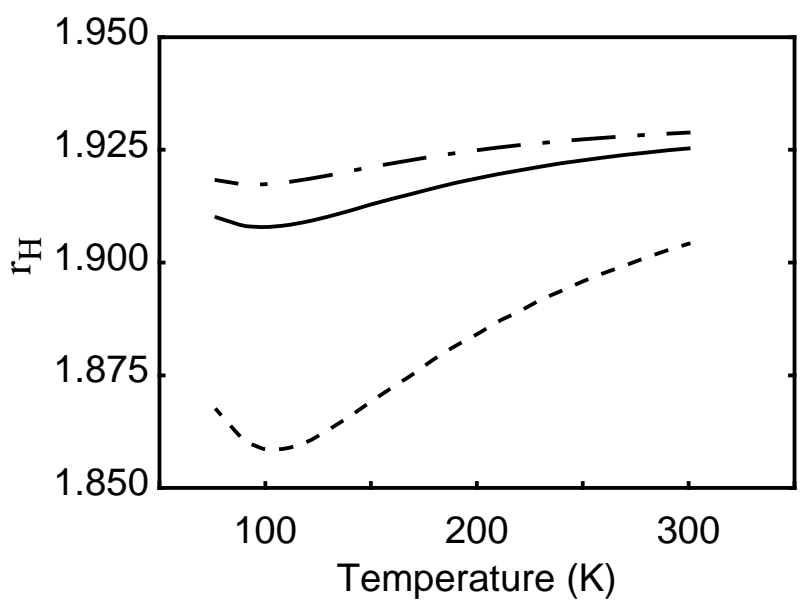

Figure 4: Hall factor resulting from dislocation scattering vs. temperature with constant donor doping densities of $5 \times 10^{16} \mathrm{~cm}^{-3}$ (dash-dotted), $10^{17} \mathrm{~cm}^{-3}$ (solid), and $5 \times 10^{17} \mathrm{~cm}^{-3}$ (dashed).

\section{REFERENCES}

${ }^{1}$ D.C. Look and R.J. Molnar, Appl. Phys. Lett., 70, 3377 (1997).

${ }^{2}$ N.G. Weimann, L.F. Eastman, D. Doppalapudi, H.M. Ng, and T.D. Moustakas, J. Appl. Phys., 83, 3656 (1998).

${ }^{3}$ R.J. Molnar, T. Lei, and T.D. Moustakas, Appl. Phys. Lett., 62, 72 (1993).

${ }^{4}$ W. Götz, L.T. Romano, J. Walker, N.M. Johnson, and R.J. Molnar, Appl. Phys. Lett., 72, 1214 (1998).

${ }^{5}$ W. Götz, N.M. Johnson, C. Chen, H. Liu, and W. Imler, Appl. Phys. Lett., 68, 3144 (1996).

${ }^{6}$ A.D. Boardman, W. Fawcett, and J.G. Ruch, Phys. Stat. Sol., 4, 133 (1971).

${ }^{7}$ J.E. Dijkstra and W. Th. Wenckebach, Appl. Phys. Lett., 70, 2428 (1997).

${ }^{8}$ P. Warmenbol, F.M. Peeters, and J.T. DeVreese, Phys. Rev. B, 33, 1213 (1986).

${ }^{9}$ C.K. Williams, H.A. Littlejohn, T.H. Glisson, and J.R. Hauser, Superlattices and Microstructures, 2, 201 (1986).

${ }^{10}$ J.D. Albrecht, R.P. Wang, P.P. Ruden, M. Farahmand, and K.F. Brennan, J. Appl. Phys., 83, 4777 (1998).

${ }^{11}$ R.P. Wang, P.P. Ruden, J. Kolnik, I. Oguzman, and K.F. Brennan, Mat. Res. Soc. Symp. Proc., 445, 935 (1997).

${ }^{12}$ J.R. Meyer and F.J. Bartoli, Phys. Rev. B, 24, 2089 (1981).

${ }^{13}$ See, e.g., L. Schiff, Quantum Mechanics, McGraw-Hill, New York (1955).

${ }^{14}$ For a canonical list of analytical relaxation-time approximations see D.C. Look, Electrical Characterization of GaAs Materials and Devices, John Wiley \& Sons, New York (1989).

${ }^{15}$ D.C. Look, J.R. Sizelove, S. Keller, Y.F. Wu, U.K. Mishra, and S.P. DenBaars, Solid State Commun., 102, 297 (1997).

${ }^{16}$ A.E. Wickenden, D.K. Gaskill, D.D. Koleske, K. Doverspike, D.S. Simons, and P.H. Chi, Mat. Res. Soc. Symp. Proc., 395, 679 (1996).

${ }^{17}$ H.M. Ng, D. Doppalapudi, T.D. Moustakas, N.G. Weimann, and L.F. Eastman, Appl. Phys. Lett., 73, 821 (1998). 